\title{
Evaluation of Rapid Testing Algorithms for Venue-based Anonymous HIV Testing among Non-HIV-Positive Men Who Have Sex with Men, National HIV Behavioral Surveillance (NHBS), 2017
}

\author{
Shamaya Whitby ${ }^{1}$ (D) Amanda Smith ${ }^{2} \cdot$ Rebecca Rossetti $^{2} \cdot$ Johanna Chapin-Bardales $^{2} \cdot$ Amy Martin $^{2}$. \\ Cyprian Wejnert ${ }^{2} \cdot$ Silvina Masciotra ${ }^{2} \cdot$ for the NHBS Study Group
}

Published online: 10 July 2020

(c) The Author(s) 2020

\begin{abstract}
HIV rapid testing algorithms (RTAs) using any two orthogonal rapid tests (RTs) allow for on-site confirmation of infection. RTs vary in performance characteristics therefore the selection of RTs in an algorithm may affect identification of infection, particularly if acute. National HIV Behavioral Surveillance (NHBS) assessed RTAs among men who have sex with men recruited using anonymous venue-based sampling. Different algorithms were evaluated among participants who self-reported never having received a positive HIV test result prior to the interview. NHBS project areas performed sequential or parallel RTs using whole blood. Participants with at least one reactive RT were offered anonymous linkage to care and provided a dried blood spot (DBS) for testing at CDC. Discordant results (RT-1 reactive/RT-2 non-reactive) were tested at CDC with lab protocols modified for DBS. DBS were also tested for HIV-1 RNA (VL) and antiretroviral (ARV) drug levels. Of 6500 RTAs, 238 were RT-1 reactive; of those, 97.1\% (231/238) had concordant results (RT-1/RT-2 reactive) and 2.9\% (7/238) had discordant results. Five DBS associated with discordant results were available for confirmation at CDC. Four had nonreactive confirmatory test results that implied RT-1 false reactivity; one had ambiguous confirmatory test results which was non-reactive in further testing. Regardless of order and type of RT used, RTAs demonstrated high concordant results in the population surveyed. Additional laboratory testing on DBS following discordant results confirmed no infection. Implementing RTAs in the context of anonymous venue-based HIV testing could be an option when laboratory follow-up is not practicable.
\end{abstract}

Keywords HIV rapid testing · HIV rapid tests algorithm · False-reactivity · Discordant RT result

\section{Introduction}

Nonclinical testing settings allow HIV testing programs to reach populations at increased risk who otherwise might not engage in regular HIV testing. Confirmation of HIV

Shamaya Whitby

lvi3@cdc.gov

Amanda Smith

zbp9@cdc.gov

Rebecca Rossetti

nvp4@cdc.gov

Johanna Chapin-Bardales

wif3@cdc.gov

Amy Martin

zhp6@cdc.gov

Cyprian Wejnert

dwy7@cdc.gov infection in nonclinical settings can expedite linkage to care for newly infected persons, those unaware of infection, and persons previously diagnosed but not in care [1,2]. The 2016 guidelines published for point-of-care (POC) HIV testing in nonclinical settings highlighted three possible testing

Silvina Masciotra

svm6@cdc.gov

1 Oak Ridge Institute for Science and Education at the Division of HIV/AIDS Prevention, National Center for HIV/AIDS, Viral Hepatitis, Division of HIV/AIDS Prevention, National Center for HIV/AIDS, Viral Hepatitis, STD and TB Prevention, Centers for Disease Control and Prevention, 1600 Clifton Rd NE MS-A25, Atlanta, GA 30329, USA

2 Division of HIV/AIDS Prevention, National Center for HIV/AIDS, Viral Hepatitis, STD and TB Prevention, Centers for Disease Control and Prevention, 1600 Clifton RdNE, Atlanta, GA 3033, USA 
algorithms: (1) a single rapid test with immediate linkage to clinical provider if initial test is reactive, (2) a single rapid test followed by laboratory-based follow-up testing if initial test is reactive, or (3) a rapid test immediately followed by a second rapid test on-site if the initial test is reactive [2-4]. We evaluated the third nonclinical testing algorithm within National HIV Behavioral Surveillance (NHBS), which conducts anonymous cross-sectional behavioral surveys and HIV testing in annual rotating cycles with men who have sex with men (MSM), persons who inject drugs, and heterosexually active persons at increased risk for HIV. In 2017, NHBS recruited a large sample of MSM using venue-based sampling in 23 U.S. metropolitan statistical areas [5, 6].

MSM are a population at increased risk for HIV acquisition who could benefit from access to low-barrier HIV testing. In the 2014 NHBS MSM survey, one in five MSM were HIV positive and one in four who were positive were potentially unaware of their infection [5]. Until 2017, NHBS participants received one RT and, if reactive or preliminary positive, confirmation was performed using laboratorybased HIV IgG/IgM or $\mathrm{Ag} / \mathrm{Ab}$ immunoassays at local or CDC laboratories. This created a barrier to knowledge of confirmed HIV status as participants needed to call or return in 1-2 weeks for confirmatory results [7]. In the U.S., an estimated $25-48 \%$ of participants who receive conventional HIV laboratory testing do not return for final confirmatory test results $[8,9]$. A meta-analysis found that participants who received a RT were 1.5-2.2-times more likely to obtain their test results than those who received standard laboratory testing [10].

As indicated in 2016 guidelines for non-clinical settings, a rapid-rapid testing algorithm (RTA) allows participants to receive their results the same day that testing is performed. In NHBS, the use of a RTA was implemented in 2017 to replace laboratory confirmatory testing [7, 11]. Although the NHBS recommendation was to include two orthogonal RTs, the order (sequential or parallel) in which each RT was performed was not specified. For this analysis, the first RT performed in an RTA we define as RT-1 and the second as RT-2. If RTs were performed in parallel, RT-1 refers to the first RT conducted. Given that false-positive results are possible, employing an RTA allows for establishing concordance between the two tests (RT-1 reactive/RT-2 reactive) or whether they were discordant (RT-1 reactive/RT-2 non-reactive). Laboratory testing was included to address RT false-reactivity. This analysis evaluated the ability of different RTAs in non-clinical settings to provide confirmation of HIV infection status by calculating the frequency of concordant results and false-reactive results.

\section{Methods}

\section{NHBS Survey}

During 2017, MSM were recruited for NHBS using venuebased, time-space sampling at places such as clubs, bars, and street locations that are frequented by MSM [12]. Project areas used an anonymous standardized questionnaire to collect information on HIV-related risk behavior, the use of prevention services, and HIV testing, following national protocols with adaptations for study populations and local policies [13, 14]. Voluntary anonymous HIV testing was offered with counseling and anonymous linkage to care services. Based on their self-reported HIV status during the survey, participants were classified as either HIV-positive or non-HIV-positive. Self-reported non-HIV-positive $(\mathrm{SRNH}+)$ participants were defined as reporting never having received a positive HIV test result. This included participants who never received an HIV test or received an HIV test but had a negative or unknown test result or never received the test result.

\section{NHBS HIV Testing and Data Collection}

NHBS project areas could have implemented a sequential RTA (s-RTA) involving two different orthogonal RTs conducted in sequence or a parallel RTA (p-RTA) in which two orthogonal RTs were conducted simultaneously. Orthogonal tests were either a similar kind of test but from two different manufacturers or two tests from the same manufacturer that relied on different principles of detection [6, 15]. NHBS project areas chose the RTs they used and the test order in their adopted algorithms; the tests used were any combination of INSTI ${ }^{\circledR}$ HIV-1/HIV-2 Antibody Test (INSTI; BioLytical, Richmond, BC), Alere Determine $^{\mathrm{TM}} \mathrm{HIV}-1 / 2 \mathrm{Ag} / \mathrm{Ab}$ Combo (DC; Alere Scarborough, Scarborough, ME), Uni-Gold ${ }^{\mathrm{TM}}$ Recombigen ${ }^{\circledR}$ HIV1/2 (Unigold; Trinity Biotech, Bray, Ireland), Chembio SURE CHECK® HIV 1/2 Assay (Sure Check; Chembio Diagnostic Systems, Inc, Medford, NY), and OraQuick ADVANCE® Rapid HIV-1/2 Antibody Test (OraQuick; OraSure Technologies, Bethlehem, Pennsylvania). Eighteen project areas followed a s-RTA, one project area performed a p-RTA, and four project areas chose to use one RT followed by laboratory confirmation. Local laboratory confirmatory HIV testing was done using Avioq HIV-1 Microelisa System (Avioq, Inc., Rockville, MD) and/or HIV-1 Western Blot (WB; OraSure Technologies, 
Bethlehem, Pennsylvania: Bio-Rad Laboratories, Hercules, California) with DBS specimens or Abbott ARCHITECT HIV Ag/Ab Combo (Abbott Laboratories, Wiesbaden, Germany) with plasma specimens. Confirmation at CDC lab was performed using GS HIV Combo $\mathrm{Ag}$ / Ab EIA (BRC; Bio-Rad Laboratories) and Geenius HIV$1 / 2$ supplemental assay (Geenius; Bio-Rad Laboratories) with DBS specimens. In project areas that implemented a s-RTA, all participants received a RT-1 and, if reactive, a RT-2 was performed. If the participant had any reactive RT and consented to blood storage, a DBS card was made from either finger-stick or venous whole blood for storage and future testing at CDC. In project areas that implemented a p-RTA, all participants received both RT-1 and RT- 2 concurrently and DBS was also collected from participants that had any reactive RT and consented to blood storage. DBS were collected following a standard protocol, dried for a minimum of $4 \mathrm{~h}$ and up to $24 \mathrm{~h}$ at ambient temperature, and then placed in sealable bags with desiccants and a humidity indicator card [16]. The bagged DBS were stored in a dry environment at ambient temperature for up to a week before shipping to CDC. Once received DBS were inventoried and individually re-bagged with desiccants and a humidity indicator card and then stored at $-20{ }^{\circ} \mathrm{C}$ [17].

\section{Testing at CDC}

DBS samples from participants with discordant results (RT-1 reactive/RT-2 non-reactive) were tested at the CDC lab to confirm HIV infection status. CDC used previously published protocols for GS HIV Combo Ag/Ab EIA (BRC; Bio-Rad Laboratories) and Geenius HIV-1/2 supplemental assay (Geenius; Bio-Rad Laboratories) which were validated for use with DBS in the CDC laboratory [18]. All samples received viral load (VL) testing using a modified protocol (HIV-1 RNA $1.0 \mathrm{~mL}$ extraction protocol) on the Abbott RealTime m2000sp HIV-1 VL kit (Abbott Molecular Inc., Des Plaines, IL) optimized for $70 \mu \mathrm{L}$ of whole blood [19, 20]. Because DBS collected during the study may have limited quantity of blood, four $6 \mathrm{~mm}$ punches $(\sim 50 \mu \mathrm{L}$ of whole blood) were used in a laboratory-validated protocol [21]. Blood was eluted in $1.3 \mathrm{~mL}$ of mLysis buffer in master mix tubes, both provided with the Abbott $\mathrm{m}$ Sample Preparation System (m2000sp). Samples were incubated at $55^{\circ} \mathrm{C}$ for 30 min, vortexed, spun, and placed on m2000 for sample extraction and VL quantification according to the standard HIV-1 RNA quantitative assay $1.0 \mathrm{~mL}$ protocol [22].

The CDC lab also quantified concentrations of the antiretroviral drugs, Raltegravir (RAL), Tenofovir (TFV), Abacavir (ABC), Ritonavir (RTV), Lamivudine (3TC), Efavirenz
(EFV), Emtricitabine (FTC), Elvitegravir (EVG) and Dolutegravir (DTG) in DBS using high-performance liquid chromatography-tandem mass spectrometry (HPLC-MS) (Sciex, Foster City, CA, Shimadzu Scientific, Columbia, MD). Briefly, $500 \mu \mathrm{L}$ of $75 \%$ acetonitrile containing internal standards, isotopically labeled RAL, TFV, EVG and FTC (Toronto Research Chemicals Inc, Toronto, Canada), were added to one $6 \mathrm{~mm}$ punch and sonicated for $30 \mathrm{~min}$ to extract drugs. The supernatant was moved to a 96-well plate, evaporated to near dryness and re-suspended in $150 \mu \mathrm{L}$ of mobile phase A $(0.2 \%$ formic acid in water). Ten $\mu \mathrm{L}$ of the final solution was injected onto a UK-C18 column $(100 \times 1 \mathrm{~mm}$, Imtakt, Portland, OR) connected to an HPLC-MS/MS system [23]. An aqueousacetonitrile mobile-phase gradient was used to elute the drugs from the column and into the analyzer. Two mass transitions $(\mathrm{Q} 1 \longrightarrow \mathrm{Q} 3)$ (where possible) were monitored in positive MRM (Multiple Reaction Monitoring) mode which were as follows: RAL- $445.00 \mathrm{~m} / \mathrm{z} \longrightarrow 109.10 \mathrm{~m} / \mathrm{z}$ and $445.00 \mathrm{~m} /$ $\mathrm{z} \longrightarrow 361.20 \mathrm{~m} / \mathrm{z} ;$ TFV- $288.00 \mathrm{~m} / \mathrm{z} \longrightarrow 176.30 \mathrm{~m} / \mathrm{z}$ and $288.00 \mathrm{~m} / \mathrm{z} \longrightarrow 159.10 \mathrm{~m} / \mathrm{z} ;$ ABC- $287.20 \mathrm{~m} / \mathrm{z} \longrightarrow 153.10 \mathrm{~m} / \mathrm{z}$ and $287.20 \mathrm{~m} / \mathrm{z} \longrightarrow 191.00 \mathrm{~m} / \mathrm{z} ; 3$ TC $-230.00 \mathrm{~m} / \mathrm{z}$ $\longrightarrow 111.90 \mathrm{~m} / \mathrm{z} ;$ EFV- $316.10 \mathrm{~m} / \mathrm{z} \longrightarrow 244.20 \mathrm{~m} / \mathrm{z}$ and $316.10 \mathrm{~m} / \mathrm{z} \longrightarrow 168.00 \mathrm{~m} / \mathrm{z} ;$ FTC- $248.00 \mathrm{~m} / \mathrm{z} \longrightarrow 130.10 \mathrm{~m} / \mathrm{z}$ and $248.00 \mathrm{~m} / \mathrm{z} \longrightarrow 113.10 \mathrm{~m} / \mathrm{z} ;$ RTV-721.20 m/ $\mathrm{z} \longrightarrow 296.20 \mathrm{~m} / \mathrm{z}$ and $721.20 \mathrm{~m} / \mathrm{z} \longrightarrow 268.10 \mathrm{~m} / \mathrm{z}$; EVG$448.20 \mathrm{~m} / \mathrm{z} \longrightarrow 344.10 \mathrm{~m} / \mathrm{z}$ and $448.20 \mathrm{~m} / \mathrm{z} \longrightarrow 143.10 \mathrm{~m} / \mathrm{z}$; and DTG-420.30 m/z $\longrightarrow 277.20 \mathrm{~m} / \mathrm{z}$ and $420.30 \mathrm{~m} /$ $\mathrm{z} \longrightarrow 127.10 \mathrm{~m} / \mathrm{z}$, respectively. The drug concentrations were estimated from a standard curve with a range of 0.5-2000 ng/ $\mathrm{mL}$ constructed using spiked blood to make DBS of appropriate concentrations [24] and analyzed using Analyst software (Sciex, Foster City, CA). The lower limit of quantification (LOQ) for all analytes in this assay was between 10 and $25 \mathrm{ng} / \mathrm{mL}$. The detectable concentrations were measured and reported in the ranges $>500 \mathrm{ng} / \mathrm{mL}(+++), 51-500 \mathrm{ng} / \mathrm{mL}$ $(++), \mathrm{LOQ}-50 \mathrm{ng} / \mathrm{mL}(+)$ with the approximate days since last dose.

\section{SAS Analysis}

This analysis was limited to non-HIV-positive participants in the 19 NHBS project areas that performed an s-RTA or p-RTA. The four project areas that performed one RT followed by local confirmation were excluded. Participants were eligible if they consented to the survey and provided valid, complete responses, consented to HIV testing, completed the s-RTA or p-RTA, had valid test results, and self-reported being non-HIV-positive. Only participants who further consented to DBS storage were included in analyses of CDC laboratory test results. Numbers and frequencies of rapid HIV test results by RTAs were calculated using SAS 9.4 (SAS Institute, Cary, NC). 


\section{Results}

During the 2017 MSM data collection, 11,232 participants were screened to participate in the 19 (18 s-RTA project areas and 1 p-RTA project area) NHBS project areas included in this analysis. Of the 6500 eligible participants, 6182 received a s-RTA while 318 received a p-RTA. Of 6182 receiving a s-RTA, the frequency at which each test was used
Table 1 Variation of sequential RTAs that started with Determine Combo among nonHIV-positive men who have sex with men in 19 U.S. citiesNational HIV Behavioral Surveillance, 2017

\begin{tabular}{lllll}
\hline $\begin{array}{l}\text { Rapid testing algo- } \\
\text { rithm (RTA) }\end{array}$ & $\begin{array}{l}\text { Number of participants } \\
\text { tested according to RTA }\end{array}$ & $\begin{array}{l}\text { RT-1-Non-reactive } \\
\% \text { (fraction) }\end{array}$ & $\begin{array}{l}\text { Concordant } \\
\text { RT }(\%)^{1}\end{array}$ & $\begin{array}{l}\text { Discordant } \\
\text { RT }(\%)^{2}\end{array}$ \\
& & & $\begin{array}{l}\text { RT-1-R->RT- } \\
2-\mathrm{R} \% \text { (fraction) }\end{array}$ & $\begin{array}{l}\text { RT-1-R- }>\text { RT- } \\
\text { 2-NR \% (frac- } \\
\text { tion) }\end{array}$ \\
\hline $\mathrm{DC} \rightarrow$ INSTI & 2295 & $95.8(2198 / 2295)$ & $94.8(92 / 97)$ & $5.2(5 / 97)$ \\
$\mathrm{DC} \rightarrow$ Sure Check & 404 & $96.3(389 / 404)$ & $100(15 / 15)$ & 0 \\
$\mathrm{DC} \rightarrow$ Unigold & 126 & $97.6(123 / 126)$ & $100(3 / 3)$ & 0 \\
\hline
\end{tabular}

HIV rapid tests: DC: Alere Determine HIV-1/2 Ag/Ab Combo Rapid Test, INSTI: BioLytical INSTI HIV -1/ HIV-2 Antibody Test, Sure Check: Chembio SureCheck HIV-1/2 Assay, Unigold: Trinity Biotech Unigold Recombigen HIV-1/2

$N$ Number of nonreactive or reactive rapid test results

$(\%)^{1}$ represents the concordance percentage for each of the combinations of RTs and $(\%)^{2}$ represents the discordant percentage for each of the combinations of RTs

Table 2 Variation of sequential RTAs that started with INSTI among non-HIV-positive men who have sex with men in 19 U.S. cities-National HIV Behavioral Surveillance, 2017

\begin{tabular}{|c|c|c|c|c|}
\hline \multirow[t]{2}{*}{ Rapid testing algorithm (RTA) } & \multirow[t]{2}{*}{$\begin{array}{l}\text { Number of participants tested } \\
\text { according to RTA }\end{array}$} & \multirow[t]{2}{*}{$\begin{array}{l}\text { RT-1-Non-reactive } \% \\
\text { (fraction) }\end{array}$} & $\begin{array}{l}\text { Concordant } \\
\text { RT }(\%)^{1}\end{array}$ & $\begin{array}{l}\text { Discordant } \\
\text { RT }(\%)^{2}\end{array}$ \\
\hline & & & $\begin{array}{l}\text { RT-1-R-> RT-2-R \% } \\
\text { (fraction) }\end{array}$ & $\begin{array}{l}\text { RT-1-R-> RT- } \\
2-\mathrm{NR} \% \text { (frac- } \\
\text { tion) }\end{array}$ \\
\hline $\mathrm{INSTI} \rightarrow \mathrm{DC}$ & 1134 & $97.3(1104 / 1134)$ & $96.7(29 / 30)$ & $3.3(1 / 30)$ \\
\hline INSTI $\rightarrow$ Sure Check & 469 & $96.6(453 / 469)$ & $100(16 / 16)$ & 0 \\
\hline INSTI $\rightarrow$ Unigold & 352 & $99.7(351 / 352)$ & $100(1 / 1)$ & 0 \\
\hline
\end{tabular}

HIV rapid tests: INSTI: BioLytical INSTI HIV -1/ HIV-2 Antibody Test, DC: Alere Determine HIV-1/2 Ag/Ab Combo Rapid Test, Sure Check: Chembio SureCheck HIV-1/2 Assay, Unigold: Trinity Biotech Uni-gold Recombigen HIV-1/2.

$N$ Number of nonreactive or reactive rapid test results

$(\%)^{1}$ represents the concordance percentage for each of the combinations of RTs and $(\%)^{2}$ represents the discordant percentage for each of the combinations of RTs

Table 3 Sequential RTAs that started with Unigold or Sure Check among non-HIV-positive men who have sex with men in 19 U.S. citiesNational HIV Behavioral Surveillance, 2017

\begin{tabular}{|c|c|c|c|c|}
\hline \multirow[t]{2}{*}{ Rapid testing algorithm (RTA) } & \multirow[t]{2}{*}{$\begin{array}{l}\text { Number of participants tested } \\
\text { according to RTA }\end{array}$} & \multirow[t]{2}{*}{$\begin{array}{l}\text { RT-1-Non-Reactive } \% \\
\text { (fraction) }\end{array}$} & \multirow{2}{*}{$\begin{array}{l}\text { Concordant } \\
\text { RT }(\%)^{1} \\
\text { RT-1-R-> RT-2-R \% } \\
\text { (fraction) }\end{array}$} & \multirow{2}{*}{$\begin{array}{l}\text { Discordant } \\
\text { RT }(\%)^{2} \\
\text { RT-1-R-> RT- } \\
\text { 2-NR \% (frac- } \\
\text { tion) }\end{array}$} \\
\hline & & & & \\
\hline Unigold $\rightarrow$ INSTI & 528 & $94.3(498 / 528)$ & $100(30 / 30)$ & 0 \\
\hline SureCheck $\rightarrow$ INSTI & 851 & $96.7(823 / 851)$ & $100(28 / 28)$ & 0 \\
\hline SureCheck $\rightarrow$ OQ & 23 & $91.3(21 / 23)$ & $100(2 / 2)$ & 0 \\
\hline
\end{tabular}

Sure Check: Chembio SureCheck HIV-1/2 Assay, INSTI: BioLytical INSTI HIV -1/ HIV-2 Antibody Test, Unigold: Trinity Biotech Uni-gold Recombigen HIV-1/2, OQ: OraQuick ADVANCE Rapid HIV-1/2 Antibody Test

$N$ Number of nonreactive or reactive rapid test results

$(\%)^{1}$ represents the concordance percentage for each of the combinations of RTs and $(\%)^{2}$ represents the discordant percentage for each of the combinations of RTs 
for RT-1 was 2825 (45.7\%) for DC; 1955 (31.6\%) for INSTI; $528(8.5 \%)$ for Unigold; and 874 (14.1\%) for Sure Check. Regardless of the RT used 5960 (96.4\%) were RT-1 nonreactive and received no further testing; 222 (3.6\%) were RT-1 reactive and received a RT-2. Of the 222 RT-1 reactive, $216(97.3 \%)$ were RT-2 reactive (concordant results) and 6 (2.7\%) were RT-2 non-reactive (discordant results).

Tables 1 through 3 show the results of specific s-RTAs adopted in 18 project areas with concordance and discordance reported for each combination of RTs. Of 2825 participants testing initially with DC, 2295 (81.2\%) were tested with INSTI as a second RT, $404(14.3 \%)$ with Sure Check and 126 (4.5\%) with Unigold. There was an overall concordance of 110/115 (95.7\%) when DC was followed by any of the RTs while $5.2 \%$ participants tested with DC followed only by INSTI had discordant results (Table 1).

For the s-RTA starting with INSTI, 1955 participants were initially tested of which 1134 (58\%) participants were tested with DC as a second RT, 469 (24\%) with Sure Check, and $352(18 \%)$ with Unigold. There was an overall concordance of 46/47 (97.9\%) when INSTI was followed by DC, Sure Check, or Unigold. While 3.3\% of participants tested using INSTI followed by DC had discordant results (Table 2).

Several project areas performed other variations of a s-RTA (Table 3). Only one s-RTA started with Unigold and was followed by INSTI which 528 (100\%) participants were tested. For the s-RTA starting with Sure Check 874 participants were initially tested. Using INTSI as the second RT $851(97.4 \%)$ participants were tested and $23(2.6 \%)$ were tested with OQ. Unigold followed by INSTI and Sure Check followed by INSTI or OQ showed $100 \%$ concordance.

A p-RTA was adopted in one project area which screened 318 participants using Sure Check and OraQuick. In the
p-RTA, participants received two orthogonal RT concurrently which returned $16 \mathrm{RT}$ reactive on the first RT used. Fifteen (4.7\%) participants had concordant reactive results and $302(95 \%)$ participants had concordant nonreactive results. One $(0.3 \%)$ participant had discordant results (SureCheck reactive, OraQuick non-reactive). The overall concordance for this algorithm was $99.6 \%$.

Table 4 shows the testing performed to confirm HIV infection in samples from participants with discordant results in s-RTAs/p-RTAs. Although DC can differentiate $\mathrm{Ag} / \mathrm{Ab}$ reactivity, the individual analyte reactivity data was not collected; only the overall reactive or non-reactive results were recorded. Of the five participants (samples 1-5) with discordant results in the DC-INSTI s-RTA, three consented to DBS storage and had DBS samples sent to CDC for confirmatory testing. The BRC was nonreactive for these and, therefore, Geenius was not performed following the recommended HIV diagnostic algorithm. Undetectable VL results and ARVs in samples 1 and 2 suggested DC false reactivity. Sample 3 had $\sim 50 \mathrm{ng} / \mathrm{mL}$ of Dolutegravir (DTG) which could not exclude viral suppression. Sample 4 was HIV-1 WB antibody-negative which did not confirm the DC $\mathrm{Ag} / \mathrm{Ab}$-reactive result based on local testing, a DBS sample was not sent to CDC. Sample 5 did not receive confirmatory testing locally or at CDC lab as a sample was not received. The s-RTA starting with INSTI followed by DC had one participant with discordant results (sample 6); this sample was BRC reactive but Geenius HIV-1 Ab negative and VL and drug levels were also not detected, likely suggesting false reactivity in both the INSTI and BRC screening tests. In the p-RTA with Sure Check and OraQuick, one participant had discordant results (sample 7). This sample was tested locally and at CDC where WB, BRC, and VL results indicated Sure Check false reactivity.

Table 4 Results of confirmatory testing on dried blood spot specimens from non-HIV-positive men who have sex with men with discordant rapid test results—National HIV Behavioral Surveillance, 19 U.S. cities, 2017

\begin{tabular}{lllllll}
\hline $\begin{array}{l}\text { Sample } \\
\text { number }\end{array}$ & RT/results 1 & RT/results 2 & $\begin{array}{l}\text { Comments/local } \\
\text { testing }\end{array}$ & $\begin{array}{l}\text { Bio-Rad GS Ag/ } \\
\text { Ab Combo EIA } \\
\text { (BRC) }\end{array}$ & $\begin{array}{l}\text { Bio-Rad Geenius } \\
\text { HIV-1/2 Supple- } \\
\text { mental }\end{array}$ & $\begin{array}{l}\text { Abbott RealTime } \\
\text { HIV-1 RNA assay } \\
\text { (copies/mL) }\end{array}$ \\
\hline 1 & DC-R & INSTI-NR & Non-Reactive & Target not detected Not detected \\
2 & DC-R & INSTI-NR & & Non-Reactive & Target not detected Not detected \\
3 & DC-R & INSTI-NR & & Non-Reactive & Target not detected DTG $(+/ 4)$ \\
4 & DC-R & INSTI-NR & WB HIV-negative & & Not done \\
5 & DC-R & INSTI-NR & DBS not sent & DBS not sent & Not done \\
6 & INSTI-R & DC-NR & & $\begin{array}{l}\text { Reactive } \\
\text { (S/CO=10.1) }\end{array}$ & $\begin{array}{c}\text { HIV-1 Antibody } \\
\text { Negative }\end{array}$ & Target not detected Not detected \\
7 & Sure Check-R & OQ-NR & WB HIV-negative & Non-Reactive & & Target not detected Insufficient quantity \\
\hline
\end{tabular}

$D T G$ Dolutegravir (Tivicay) at $\sim 50 \mathrm{ng} / \mathrm{ml} ; R$ reactive; $N R$ non-reactive; $W B$ Western Blot; $S / C O$ signal cut-off is calculated by dividing the OD value by assay cut off value 


\section{Discussion}

Using two orthogonal RTs to confirm HIV infection in non-clinical settings can allow for same day return of results and expedited linkage to care. We demonstrate that the high concordance of RT results in using an RTA supports that testing with two RTs is a viable option for diagnosis in populations at increased risk when conventional laboratory testing settings might not be readily accessible. Most discordant results could be attributed to RT-1 false reactivity in five of the seven cases.

While false-reactivity in HIV RTs has been widely reported, usually leading to discordant RT results, it is not common [25]. False-reactive results can occur for many reasons including but not limited to technical issues with the test device, mislabeling, improper handling, or misinterpretation of a visually read test [26]. The s-RTA starting with DC followed by INSTI had 5\% (5/97) discordant results, INSTI followed by DC had 3.3\% (1/30) discordant results, and Sure Check/OraQuick in the p-RTA had $0.3 \%$ $(1 / 318)$ discordant results. Follow-up laboratory results suggest false-reactivity in few of the discordant samples, although results were inconclusive for others. False- reactivity was confirmed by further laboratory testing in DC followed by INSTI s-RTA (samples 1-4), although the source of the detected ARV in sample 3 remains unknown and could for instance be related to post-exposure prophylaxis. In the p-RTA, Sure Check/OraQuick (sample 7) indicate false reactivity in both screening tests after laboratory confirmation via WB was performed for one sample. VL and drug levels did not confirm reactivity in INSTI and BRC (sample 6). However, we cannot discount that low viremia and ARV drug exposure could have been missed by the assays or that the participant was an elite controller (suppressed HIV-1 RNA levels to below the limit of detection in the absence of ART) [27]. In contrast, if it were an acute infection, HIV-1 RNA levels would have been higher than the limit of quantification of our validated VL assay. In the p-RTA, Sure Check/OraQuick indicate false reactivity in both screening tests after laboratory confirmation via WB was performed for one sample.

Confirmatory testing limitations include the use of WB for confirmation after screening with DC since WB cannot detect p24 antigen. Moreover, the WB assay faces many challenges such as high rate of uncertainty, low sensitivity, long testing period, and the limitation of detection in the early stages of seroconversion, at a time when cost of the assay is increasing [28-30]. During this cycle, we did not collect the individual analyte reactivity to address the impact of Ag false-reactivity frequently reported from field studies [31]. Other FDA-approved supplemental tests are available for validated off-label use with DBS or laboratory-developed tests could be used. Another confirmatory testing limitation is the sensitivity of the labvalidated Abbott RealTime HIV-1 assay using four DBS punches $(\sim 50 \mu \mathrm{L})$, instead of $70 \mu \mathrm{L}$ whole blood, that detects $66 \%$ of $3 \log$ (copies/mL) [21]; thus participants with low viremia may have been missed.

In following the s-RTA as designed, participants who self-reported being non-HIV-positive and had a non-reactive RT-1 result only received one RT; these participants were considered HIV-negative and did not receive a second RT. These samples were not sent to CDC for testing to evaluate whether any acute infections may have been missed. While RT kits are more expensive and less sensitive than conventional blood test on serum/plasma, research suggests that RTs are more cost-effective when factoring the number of people tested who receive their results when compared to conventional testing $[32,33]$. A randomized trial at an STI clinic found that $88 \%$ of MSM preferred RT to standard blood testing and a systematic review showed that $87-97 \%$ of clients would choose a RT over standard blood testing [7, 34]. It is estimated that desirability of RTs would increase uptake of HIV testing three-fold compared to standard blood testing in community-based voluntary counseling and testing settings or when approached by HIV counselors in emergency departments [7, 35-37]. Those who self-reported being HIV-positive with a negative RT result received confirmatory testing at $\mathrm{CDC}$; these findings are being analyzed separately.

\section{Conclusion}

In 2016, approximately $40 \%$ of people who were unaware of their HIV infection status or who had been previously diagnosed but not engaged in care or treatment contributed to $80 \%$ of new HIV infections in the US [38]. Using an RTA to provide definitive results may expedite linkage to care in populations at increased risk who otherwise might not seek testing. The high concordance of RTs in both the s-RTA and p-RTA sites has shown that implementing an algorithm for testing high-risk populations in non-clinical settings effectively returns results to participants who may experience barriers to receiving confirmatory results from a laboratory. The few participants with discordant results, most confirming as HIV-negative, suggested that a two-test RTA provides a reliable indication of infection status, notwithstanding persons with acute infection. The results did not find that any particular RTA performed better than another as the sample size of each algorithm differed limiting comparisons between the RTAs. Before choosing or implementing an RTA for a setting, the characteristics and HIV prevalence of the target population, characteristics and costs of RTs, and access to lab-based testing should be considered. RTAs will 
continue to be analyzed in the upcoming cycles of NHBS to evaluate effectiveness of different RTAs used.

Acknowledgements National HIV Behavioral Surveillance participants; CDC National HIV Behavioral Surveillance Team. National HIV Behavioral Surveillance Study Group. Atlanta, GA: Pascale Wortley, Jeff Todd, David Melton; Boston, MA: Monina Klevens, Rose Doherty, Conall O'Cleirigh; Chicago, IL: Stephanie Masiello Schuette, Antonio D. Jimenez; Dallas, TX: Jonathon Poe, Margaret Vaaler, Jie Deng; Denver, CO: Alia Al-Tayyib, Melanie Mattson; Detroit, MI: Vivian Griffin, Emily Higgins, Mary-Grace Brandt; Houston, TX: Salma Khuwaja, Zaida Lopez, Paige Padgett; Los Angeles, CA: Ekow Kwa Sey, Yingbo Ma; Miami, FL: Emma Spencer, Willie Nixon, David Forrest; Nassau-Suffolk, NY: Bridget Anderson, Ashley Tate, Meaghan Abrego; New Orleans, LA: William T. Robinson, Narquis Barak, Jeremy M. Beckford; New York City, NY: Sarah Braunstein, Alexis Rivera, Sidney Carrillo Newark, NJ: Barbara Bolden, Afework Wogayehu, Henry Godette; Philadelphia, PA: Kathleen A. Brady, Chrysanthus Nnumolu, Jennifer Shinefeld; Portland, OR: Sean Schafer, E. Roberto Orellana, Amisha Bhattari; San Francisco, CA: H. Fisher Raymond, Theresa Ick; San Juan, PR: Sandra Miranda De León, Yadira Rolón-Colón; Seattle, WA: Tom Jaenicke, Sara Glick; Virginia Beach, VA: Celestine Buyu, Toyah Reid, Karen Diepstra; CDC: Monica Adams, Christine Agnew Brune, Qian An, Alexandra Balaji, Dita Broz, Janet Burnett, Johanna Chapin-Bardales, Melissa Cribbin, YenTyng Chen, Paul Denning, Katherine Doyle, Teresa Finlayson, Senad Handanagic, Brooke Hoots, Wade Ivy, Kathryn Lee, Rashunda Lewis, Evelyn Olansky, Gabriela Paz-Bailey, Taylor Robbins, Catlainn Sionean, Amanda Smith, Cyprian Wejnert, Mingjing Xia.

Disclaimer The findings and conclusions in this report are ours and do not necessarily represent the views of the Centers for Disease Control and Prevention. Use of brand names is for identification purposes and does not imply endorsements by the US Department of Health and Human Services or the Centers for Disease Control and Prevention.

ORISE Disclaimer This project was supported in part by an appointment to the Research Participation Program at the Centers for Disease Control and Prevention administered by the Oak Ridge Institute for Science and Education through an interagency agreement between the U.S. Department of Energy and the Centers for Disease Control and Prevention.

Funding None declared.

\section{Compliance with ethical standards}

Conflict of interest No financial disclosures were reported by the authors of this paper.

Open Access This article is licensed under a Creative Commons Attribution 4.0 International License, which permits use, sharing, adaptation, distribution and reproduction in any medium or format, as long as you give appropriate credit to the original author(s) and the source, provide a link to the Creative Commons licence, and indicate if changes were made. The images or other third party material in this article are included in the article's Creative Commons licence, unless indicated otherwise in a credit line to the material. If material is not included in the article's Creative Commons licence and your intended use is not permitted by statutory regulation or exceeds the permitted use, you will need to obtain permission directly from the copyright holder. To view a copy of this licence, visit http://creativecommons.org/licenses/by/4.0/.

\section{References}

1. Centers for Disease Control and Prevention (2012). Evaluation guide for HIV testing in non-clinical settings and linkage to health and prevention services. August 13, 2012 [cited 2019 June]; Available from: https://stacks.cdc.gov/view/cdc/26216.

2. Centers for Disease Control and Prevention (2016). HIV Testing in Nonclinical Settings: A Guide for HIV Testing Providers. March 2, 2016; Available from: https://www.cdc.gov/hiv/pdf/testi ng/CDC_HIV_Implementing_HIV_Testing_in_Nonclinical_Setti ngs.pdf.

3. Centers for Disease, C., Prevention, and Association of Public Health Laboratories (2014). Laboratory testing for the diagnosis of HIV infection : updated recommendations. June 27, 2014 June 27, 2014; Available from: https://stacks.cdc.gov/view/cdc/23447.

4. Association of Public Health Laboratories and Centers for Disease Control Prevention (2010). HIV Testing Algorithms; A Status Report. April 2010; Available from: https://stacks.cdc.gov/view/ cdc/5696.

5. Centers for Disease Control and Prevention (2014). HIV Infection Risk, Prevention, and Testing Behaviors among Men Who Have Sex with Men National HIV Behavioral Surveillance 20 U.S. Cities, 2014. HIV Surveillance Special Report 15 January 2016; Available from: https://www.cdc.gov/hiv/library/reports/ surveillance/\#panel2.

6. Centers for Disease Control and Prevention (2019). National HIV Behavioral Surveillance System Round 5: Model Surveillance Protocol. December 11, 2018 March 20, 2019; Available from: www.cdc.gov/hiv/statistics/systems/nhbs/operations.html.

7. Challacombe, L. B. A. L. (2015). Rapid point-of-care HIV testing: A review of the evidence Prevention in Focus. Berlin: Spring.

8. Parisi, M., et al. (2009). Offer of rapid testing and alternative biological samples as practical tools to implement HIV screening programs. New Microobiologica, 32, 391-396.

9. Constantine, N., \& Zink, H. (2005). HIV testing technologies after two decades of evolution. The Indian Journal of Medical Research, 121, 519-538.

10. Hutchinson, A. B., et al. (2004). Understanding the patient's perspective on rapid and routine HIV testing in an inner-city urgent care center. AIDS Education and Prevention, 16(2), 101-114.

11. Greenwald, J. L. (2006). Routine rapid HIV testing in hospitals: Another opportunity for hospitalists to improve care. Journal of Hospital Medicine, 1(2), 106-112.

12. Centers for Disease Control and Prevention (2019). HIV infection risk, prevention, and testing behaviors among men who have sex with men-National HIV Behavioral Surveillance, 23 U.S. Cities, 2017. February 2019, HIV Surveillance Special Report 22.

13. Finlayson, T. J., et al. (2011). HIV risk, prevention, and testing behaviors among men who have sex with men-National HIV Behavioral Surveillance System, 21 US cities United States, 2008. MMWR Surveill Summaries, 60(14), 1-34.

14. German, D., et al. (2014). Using Baltimore HIV behavioral surveillance data for local hiv prevention planning. AIDS and Behavior, 18, 359-369.

15. Selik, R. M., et al. (2014). Revised surveillance case definition for HIV infection United States, 2014. Morbidity and Mortality Weekly Report, 63(3), 1-10.

16. Curtis, K. A., et al. (2014). Evaluation of dried blood spots with a multiplex assay for measuring recent HIV-1 infection. PLOS ONE, 9(9), e107153-e107153.

17. Smith, A. J., Owen, S. M. \& Wejnert, C. for the NHBS Study Group. (2019). The Implementation of Dried Blood Spot (DBS) Collection in the Field for Supplemental HIV Testing - National HIV Behavioral Surveillance (NHBS). In 2019 HIV Diagnostics Conference 2019. Atlanta, GA. 
18. Luo, W., et al. (2017). Evaluation of dried blood spot protocols with the Bio-Rad GS HIV Combo Ag/Ab EIA and Geenius ${ }^{\text {TM }}$ HIV 1/2 Supplemental Assay. Journal of Clinical Virology, 91, 84-89.

19. Hirshfield, S., et al. (2018). Quantification of HIV-1 RNA among men who have sex with men using an at-home self-collected dried blood spot specimen: Feasibility Study. JMIR Public Health Surveillance, 4(4), e10847.

20. Tang, N., et al. (2017). HIV-1 viral load measurement in venous blood and fingerprick blood using Abbott RealTime HIV-1 DBS assay. Journal of Clinical Virology, 92, 56-61.

21. Wei, L. M. (2019). The feasibility of modified HIV and antiretroviral drug testing using self-collected dried blood spots from men who have sex with men. In 2019 HIV Diagnostics Conference 2019. Atlanta, GA.

22. Marconi, A., et al. (2009). Evaluation of the Abbott Real-Time HIV-1 quantitative assay with dried blood spot specimens. Clinical Microbiology and Infection, 15(1), 93-97.

23. Kuklenyik, Z., et al. (2009). Effect of mobile phase $\mathrm{pH}$ and organic content on LC-MS analysis of nucleoside and nucleotide HIV reverse transcriptase inhibitors. Journal of Chromatographic Science, 47(5), 365-372.

24. Mei, J. V., et al. (1998). Radioimmunoassay for monitoring zidovudine in dried blood spot specimens. Clinical Chemistry, 44(2), 281-286.

25. Centers for Disease Control and Prevention (2018). False- Positive HIV Test Results. May 2018 [cited 2019 April]; Available from: https://www.cdc.gov/hiv/pdf/testing/cdc-hiv-factsheet-false-posit ive-test-results.pdf.

26. Klarkowski, D., et al. (2014). Causes of false-positive HIV rapid diagnostic test results. Expert Review of Anti-infective Therapy, 12(1), 49-62.

27. Gunthard, H. F., et al. (2014). Antiretroviral treatment of adult HIV infection: 2014 recommendations of the International Antiviral Society-USA Panel. JAMA, 312(4), 410-425.

28. Feng, X., et al. (2017). An alternative strategy to western blot as a confirmatory diagnostic test for HIV infection. Journal of Clinical Virology, 88, 8-11.

29. Centers for Disease Control and Prevention. (2013). Detection of acute HIV infection in two evaluations of a new HIV diagnostic testing algorithm United States, 2011-2013. MMWR Morbidity and Mortality Weekly Report, 62(24), 489-494.
30. Styer, L. M., Sullivan, T. J., \& Parker, M. M. (2011). Evaluation of an alternative supplemental testing strategy for HIV diagnosis by retrospective analysis of clinical HIV testing data. Journal of Clinical Virology, 52, S35-S40.

31. Lewis, J. M., et al. (2015). Field accuracy of fourth-generation rapid diagnostic tests for acute HIV-1: A systematic review. AIDS (London, England), 29(18), 2465-2471.

32. Shrestha, R. K., et al. (2008). Cost-effectiveness of finding new HIV diagnoses using rapid HIV testing in community-based organizations. Public Health Reports, 123(Suppl 3), 94-100.

33. Farnham, P. G., et al. (2008). Comparing the costs of HIV screening strategies and technologies in health-care settings. Public Health Reports, 123(Suppl 3), 51-62.

34. de la Fuente, L., et al. (2009). Increasing early diagnosis of HIV through rapid testing in a street outreach program in Spain. AIDS Patient Care and STDs, 23(8), 625-629.

35. Walensky, R. P., et al. (2011). Counselor-versus provider-based HIV screening in the emergency department: results from the universal screening for HIV infection in the emergency room (USHER) Randomized Controlled Trial. Annals of Emergency Medicine, 58(1), S126-S132.

36. Lugada, E., et al. (2010). Comparison of home and clinic-based HIV testing among household members of persons taking antiretroviral therapy in Uganda: results from a randomized trial. Journal of Acquired Immune Deficiency Syndromes, 55(2), 245-252.

37. Sweat, M., et al. (2011). Community-based intervention to increase HIV testing and case detection in people aged 16-32 years in Tanzania, Zimbabwe, and Thailand (NIMH Project Accept, HPTN 043): a randomised study. The Lancet Infectious Diseases, 11(7), 525-532.

38. Fauci, A. S., et al. (2019). Ending the HIV epidemic: A plan for the united statesending the HIV epidemic editorial. JAMA, 321(9), $844-845$.

Publisher's Note Springer Nature remains neutral with regard to jurisdictional claims in published maps and institutional affiliations. 